\title{
ZTFJ0038+2030: A Long-period Eclipsing White Dwarf and a Substellar Companion
}

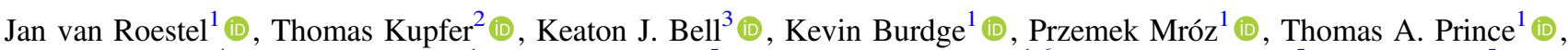 \\ Eric C. Bellm ${ }^{4}$ (D), Andrew Drake ${ }^{1}$, Richard Dekany ${ }^{5}$ (D), Ashish A. Mahabal ${ }^{1,6}$ (D), Michael Porter ${ }^{5}$, Reed Riddle ${ }^{5}$, \\ Kyung Min Shin $^{7}$ (D), David L. Shupe ${ }^{8}$ (D), and S. R. Kulkarni ${ }^{1}$ (D) \\ ${ }^{1}$ Division of Physics, Mathematics, and Astronomy, California Institute of Technology, Pasadena, CA 91125, USA; jvanroes@caltech.edu \\ ${ }^{2}$ Department of Physics and Astronomy, Texas Tech University, PO Box 41051, Lubbock, TX 79409, USA \\ ${ }^{3}$ DIRAC Institute, Department of Astronomy, University of Washington, Seattle, WA-98195, USA \\ ${ }^{4}$ DIRAC Institute, Department of Astronomy, University of Washington, 3910 15th Avenue NE, Seattle, WA 98195, USA \\ ${ }^{5}$ Caltech Optical Observatories, California Institute of Technology, Pasadena, CA 91125, USA \\ ${ }^{6}$ Center for Data Driven Discovery, California Institute of Technology, Pasadena, CA 91125, USA \\ ${ }^{7}$ California Institute of Technology, Pasadena, CA 91125, USA \\ ${ }^{8}$ IPAC, California Institute of Technology, 1200 E. California Blvd., Pasadena, CA 91125, USA \\ Received 2021 May 24; revised 2021 September 1; accepted 2021 September 1; published 2021 October 1
}

\begin{abstract}
In a search for eclipsing white dwarfs using the Zwicky Transient Facility lightcurves, we identified a deep eclipsing white dwarf with an orbital period of $10.4 \mathrm{hr}$ and an undetected substellar companion. We obtained highspeed photometry and radial velocity measurements to characterize the system. The white dwarf has a mass of $0.50 \pm 0.02 M_{\odot}$ and a temperature of $10,900 \pm 200 \mathrm{~K}$. The companion has a mass of $0.059 \pm 0.004 M_{\odot}$ and is a brown dwarf. It has a radius of $0.0783 \pm 0.0013 R_{\odot}$, and is one of the physically smallest transiting brown dwarfs known and likely old, $\gtrsim 8$ Gyr. The ZTF discovery efficiency of substellar objects transiting white dwarfs is limited by the number of epochs and as ZTF continues to collect data we expect to find more of these systems.
\end{abstract}

Unified Astronomy Thesaurus concepts: White dwarf stars (1799); Eclipsing binary stars (444); Brown dwarfs (185); Extrasolar gaseous giant planets (509)

\section{Introduction}

Low-mass, hydrogen-rich objects (brown dwarfs and giant planets) that orbit a white dwarf in short orbital periods are rare. There are currently 10 confirmed white dwarfs with brown dwarf companions with measured orbital periods. These are: GD1400 $(P=9.98 \mathrm{hr}$; Farihi \& Christopher 2004; Dobbie et al. 2005; Burleigh et al. 2011), WD0137-349 ( $P=116$ minutes; Burleigh et al. 2006; Maxted et al. 2006; Casewell et al. 2015), NLTT $5306(P=101.9$ minutes, Steele et al. 2013), WD0837+185 $(P=4.2 \mathrm{hr}$; Casewell et al. 2012), SDSS J141126.20+200911.1 $(P=121.73$ minutes; Beuermann et al. 2013; Littlefair et al. 2014), SDSS J155720.77+091624.6 $(P=2.27 \mathrm{hr}$; Farihi et al. 2017), SDSS J120515.80-024222.6 $(P=71.2$ minutes; Parsons et al. 2017a), SDSSJ123127.14+004132.9 $(P=72.5$ minutes; Parsons et al. 2017a), EPIC212235321 ( $P=68.2$ minutes; Casewell et al. 2018) and WD1032+011 $(P=2.21 \mathrm{hr}$; Casewell et al. 2020a). Most of these systems have short orbital periods. This is a detection bias: they have a higher eclipse probability, stronger reflection effect, and the white dwarf has a higher radial-velocity amplitude. For example, confirmation of GD1400 as a binary requires the white dwarf radial velocity to be measured, which requires a large telescope and high-resolution spectrograph, even for a system as bright $(G=15.2)$ as GD1400 (Burleigh et al. 2011).

There have also been many searches to find exoplanets $\left(M \lesssim 13 M_{\text {jup }}\right)$ orbiting white dwarfs, e.g., Faedi et al. (2011), Fulton et al. (2014), van Sluijs \& Van Eylen (2018), Dame et al. (2019), Rowan et al. (2019), but none of them found any candidates. The first white dwarf with a Jupiter-mass companion, WD $1145+017$, which has an orbital period of $\approx 10$ days, was only recently discovered by Gänsicke et al. (2019). The planet is slowly evaporating and this material is

\footnotetext{
NSF Astronomy and Astrophysics Postdoctoral Fellow
}

accreted by the white dwarf. Soon after, Vanderburg et al. (2020) discovered the first transiting giant planet orbiting a white dwarf, WD J0914+1914, which has an orbital period of 1.4 days. After carefully analyzing optical and infrared lightcurves of the grazing eclipse, they conclude that the companion is a giant planet with a mass of $\lesssim 14 M_{\text {jup }}$.

Because the white dwarf went through a giant phase in the past, the companion must have migrated or have been formed after the giant phase. For brown dwarfs, common-envelope evolution is the commonly accepted scenario (Ivanova et al. 2013). If a brown dwarf is close enough to a star that is ascending the red giant branch (RGB) or asymptotic giant branch (AGB) $\left(\approx 200-1000 R_{\odot}, 1-5 \mathrm{au}\right)$, the giant star engulfs the brown dwarf and forms a common envelope. Depending on the masses and initial orbital separation, the system can survive this process and ends up as a short-period binary (Casewell et al. 2018, 2020a, 2020b). However, the binary can also merge during a common-envelope event or the companion gets evaporated as soon as the hot white dwarf emerges from the common envelope (Nelemans \& Tauris 1998; Soker 1998; Bear \& Soker 2011). Particularly lower-mass objects (giant planets) are expected to merge or evaporate during this process. To form white dwarf-planet systems, alternative pathways have been proposed. These are the formation of secondgeneration planets from gas around the white dwarf (e.g., Perets 2010) or capture and/or inward migration of distant planets (e.g., Stephan et al. 2020). In addition, recent work suggests that giant planets can survive common-envelope evolution (Lagos et al. 2021).

With the goal of studying the population of white dwarfs with brown dwarf or giant planet companions in close orbits, we searched for deep eclipsing white dwarfs using Zwicky Transient Facility lightcurves (Bellm et al. 2019; Graham et al. 2019; Masci et al. 2019; Dekany et al. 2020). We used the 
Table 1

Observational Properties of ZTFJ0038+2030

\begin{tabular}{|c|c|}
\hline R.A. & $00^{\mathrm{h}} 38^{\mathrm{m}} 55^{\mathrm{s}} .0$ \\
\hline Decl. & $20^{\circ} 30^{\prime} 26^{\prime \prime} 1$ \\
\hline parallax & $7.19 \pm 0.11 \mathrm{mas}$ \\
\hline distance & $138.3_{-1.9}^{+1.7} \mathrm{pc}$ \\
\hline$E_{B-V}$ & $0.03 \pm 0.02$ \\
\hline$G^{V}$ & 17.70 \\
\hline $\mathrm{BP}^{V}$ & $17.76 \pm 0.01$ \\
\hline $\mathrm{RP}^{V}$ & $17.63 \pm 0.01$ \\
\hline GALEX FUV & $20.37 \pm 0.24$ \\
\hline GALEX NUV & $18.58 \pm 0.06$ \\
\hline ZTF- $g$ & $17.70 \pm 0.02$ \\
\hline ZTF- $r$ & $17.78 \pm 0.03$ \\
\hline $\mathrm{ZTF}-i$ & $17.95 \pm 0.03$ \\
\hline PS- $g$ & $17.705 \pm 0.005$ \\
\hline PS- $r$ & $17.786 \pm 0.002$ \\
\hline PS- $i$ & $17.931 \pm 0.006$ \\
\hline PS- $z$ & $18.093 \pm 0.005$ \\
\hline PS- $y$ & $18.18 \pm 0.02$ \\
\hline UKIRT- $J^{V}$ & $17.71 \pm 0.06$ \\
\hline WISE- $W 1^{V}$ & $17.87 \pm 0.11$ \\
\hline WISE- $W 2^{V}$ & $17.63 \pm 0.29$ \\
\hline
\end{tabular}

Note. Gaia eDR3 data were used (Brown et al. 2020b) with the geometric distance from Bailer-Jones et al. (2021). " $V$ " indicates that the magnitudes are in the Vega system; other magnitudes are in the AB system.

combined PSF photometry and alert photometry lightcurves of white dwarfs (Gentile Fusillo et al. 2019) using the box least squares algorithm (Kovács et al. 2002). For more details, see van Roestel et al. (2021).

In this paper, we present the first result, the discovery of ZTFJ003855.0+203025.5 (ZTFJ0038+2030 see Table 1), an eclipsing white dwarf with a brown dwarf companion with an orbital period of $10 \mathrm{hr}$. ZTFJ0038+2030 shows a complete eclipse in the ZTF $g$ and $r$ band with a short eclipse duty cycle. It also showed no excess luminosity in the Gaia observational $\mathrm{H}-\mathrm{R}$ diagram and no infrared excess in Pan-STARRS- $y$ or WISE $W 1$ and $W 2$, which indicates the companion is cold and either a brown dwarf or giant planet and not an $M$ dwarf. Because of these properties, we prioritized it for follow-up observations to determine the nature of the companion.

We obtained follow-up photometry and spectroscopy (Section 2), which we used the characterize the system (Section 3). We present the mass, radius, and temperature measurements in Section 4. We compare this binary system with other white dwarfs with substellar companions, and discuss the implications of this discovery for future searches for giant exoplanets around white dwarfs with ZTF (Section 5). The final section summarizes the paper.

\section{Follow-up Data}

\subsection{CHIMERA Fast Cadence Photometry}

We obtained high-speed photometry in the $g$ and $z$ filters using CHIMERA (see Table 2). CHIMERA (Harding et al. 2016) is a dual-channel photometer that uses frame-transfer, electron-multiplying CCDs mounted on the Hale 200 inch $(5.1 \mathrm{~m})$ Telescope at Palomar Observatory (CA, USA). The pixel scale is 0 ". 28 pixel $^{-1}$ (unbinned). We used the conventional amplifier and used $2 \times 2$ binning on most nights to reduce the readout noise. Each of the images was bias-subtracted and divided by twilight flat fields. We used the ULTRACAM pipeline to do aperture photometry (Dhillon et al. 2007). We used an optimal extraction method with a variable aperture of 1.5 the FWHM of the seeing (as measured from the reference star). A differential lightcurve was created by simply dividing the counts of the target by the counts from the reference star. Timestamps of the images were determined using a GPS receiver.

\subsection{ESI}

We used the Echellete Spectrograph and Imager (ESI, Sheinis et al. 2002) mounted at Keck II to obtain mediumresolution spectra $(R \approx 6000)$. The wavelength range is from 4000 to $10000 \AA$. CuAr arc exposures were taken at the beginning of the night. The spectra were reduced using the $M A K E E^{10}$ pipeline following the standard procedure: bias subtraction, flat-fielding, sky subtraction, order extraction, and wavelength calibration. We did not attempt to flux-calibrate the spectra.

\subsection{Archival Photometry}

To be able to study the spectral energy distribution, we obtained photometry data from multiple other survey telescopes (see Table 1): UV data from GALEX (Bianchi et al. 2017), optical data from Gaia eDR3 (Brown et al. 2020a) and Pan-STARRS (Chambers et al. 2016), UKIRT Hemisphere Survey J-band data (Dye et al. 2018), and far-infrared data from WISE (Marocco et al. 2021). No near-infrared photometry $H$ and $K$ data are available. We used zero-points for each of the filters to convert the magnitudes to a flux.

\section{Analysis}

\subsection{Ephemeris}

We determine the ephemeris by measuring the mid-eclipse time from the CHIMERA $g$ lightcurve. We then use the best model from the Chimera $g$ data and use it to fit all ZTF data. In addition, we noticed that there is one non-detection on 2012 November 01 in Palomar Transient Factory data (out of 94 observations). We add this epoch with half the eclipse duration as uncertainty as a prior $\left(\mathrm{BJD}_{\mathrm{TDB}}=2,456,232.8854 \pm\right.$ 0.0018). This results in an ephemeris of:

$$
\mathrm{BJD}(\mathrm{TDB})=2,459,045.985194(2)+0.4319208(14)
$$

\subsection{Spectral Energy Distribution}

To determine the white dwarf and companion temperature, we fit the observed spectral energy distribution with a model that combines white dwarf spectral models with spectral models of substellar companions (see Figure 1). We use a grid of DA white dwarf models by Koester (2009) and use bilinear interpolation to be able to generate a model for any temperature and surface gravity value. For the companion, we use models from Phillips et al. (2020) with $\log g=5.5$ and a fixed radius based on the lightcurve result. We use the extinction law by Fitzpatrick (1999) to account for any dust extinction. To compare the model spectra with the data, we convolve the

\footnotetext{
${ }^{10}$ http://www.astro.caltech.edu $\sim$ tb/ipac_staff/tab/makee/
} 


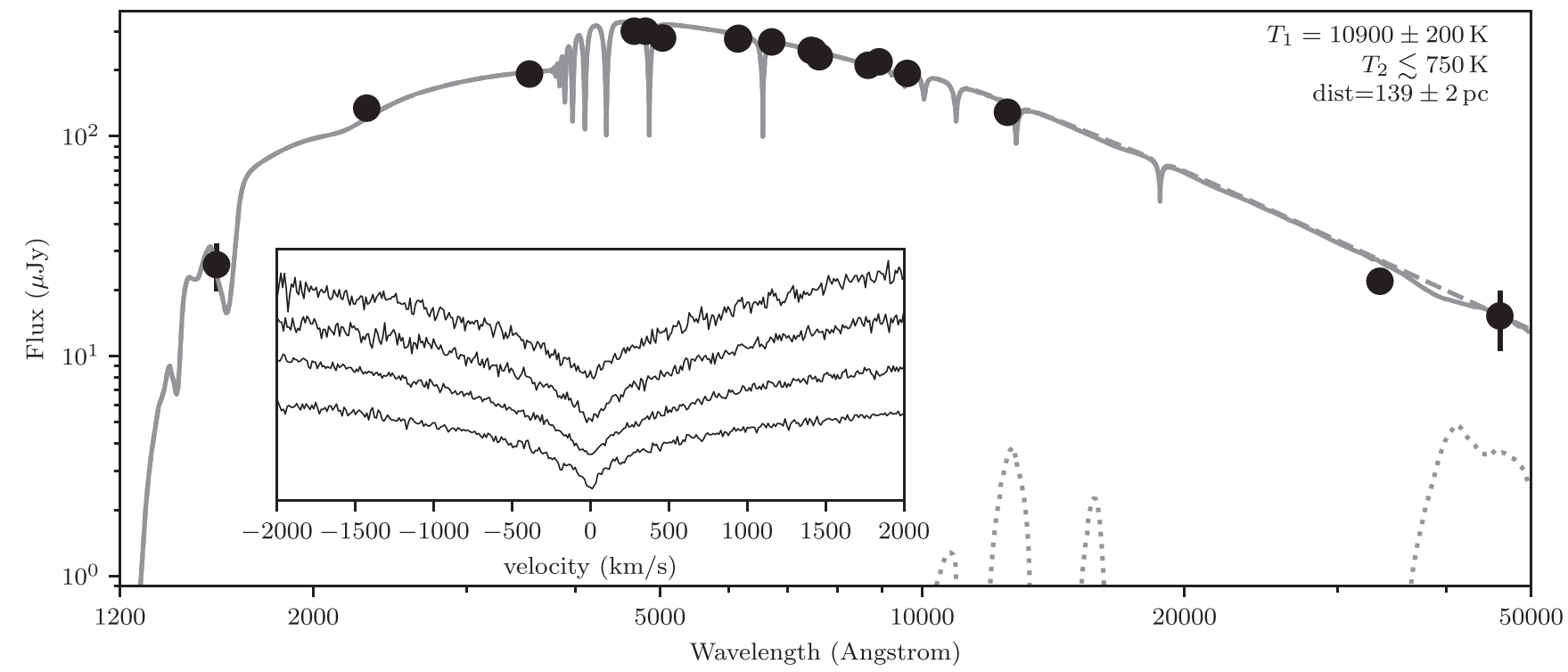

Figure 1. The spectral energy distribution of the ZTFJ0038+2030. Markers show GALEX, Gaia DR3, Pan-STARRS, median ZTF gri, and WISE data. The best-fit model is shown in gray, with the largest possible contribution by the companion as a dotted line. The substellar companion does not contribute significantly, even in the WISE $W 1$ and $W 2$ bands in the infrared. We can therefore only derive an upper limit to the companion temperature. The inset shows the averaged and velocitycorrected ESI spectra, showing from top to bottom: $\mathrm{H} \delta, \mathrm{H} \gamma, \mathrm{H} \beta$, and $\mathrm{H} \alpha$.

Table 2

Summary of the Follow-up Observations

\begin{tabular}{lcccrr}
\hline \hline Date & UT & Tele./Inst. & $N_{\text {exp }}$ & Exp. time (s) & Wavelength \\
\hline $2020-07-15$ & $11: 22-11: 57$ & P200/CHIMERA & 400 & 5.0 & 5.0 \\
$2020-07-15$ & $11: 22-11: 57$ & P200/CHIMERA & 400 & 600 & $z$ \\
$2020-07-21$ & $12: 18-12: 39$ & Keck/ESI/Echellete & 2 & 600 & $4000-10000 \AA$ \\
$2020-09-12$ & $12: 17-12: 38$ & Keck/ESI/Echellete & 2 & 600 \\
$2020-09-12$ & $14: 16-14: 37$ & Keck/ESI/Echellete & 3 & $4000-10000 \AA$ \\
\hline
\end{tabular}

model spectra with the filter response curves ${ }^{11}$ (Rodrigo et al. 2012; Rodrigo \& Solano 2020). We use Gaussian priors on the parallax using the Gaia eDR3 data, the radius estimate from the lightcurve, and an $E_{\mathrm{BV}}$ value from Pan-STARRS extinction estimates (Green et al. 2018). We again use emcee to estimate the best-fit values and uncertainties.

\subsection{Spectra and Radial Velocity Amplitude}

The spectra show a typical DA white dwarf spectrum with broad Balmer absorption lines (see the inset in Figure 1). No features from the substellar companion can be seen. There are also no Balmer emission lines due to irradiation (e.g., Parsons et al. 2018), but these are not expected to be significant given the white dwarf temperature and relatively long orbital separation.

Radial velocities of the ESI spectra were measured by fitting a Gaussian, Lorentzian, and polynomials to the $\mathrm{H} \alpha-\mathrm{H} \delta$ lines to cover the continuum, line, and line core of the individual lines using the FITSB2 routine (Napiwotzki et al. 2004). The procedure is described in full detail in Kupfer et al. (2020, 2017a, 2017b). We fitted the wavelength shifts compared to the rest wavelengths using a $\chi^{2}$ minimization.

To determine the radial-velocity semi-amplitude of the white dwarf $\left(K_{1}\right)$, we fit the radial-velocity measurements using a sinusoid with a fixed period and zero phase based on the

\footnotetext{
${ }^{11}$ http://svo2.cab.inta-csic.es/theory/fps/
}

ephemeris determined from the ZTF data. The two remaining free parameters are the amplitude $\left(K_{1}\right)$ and a systematic velocity $(\gamma)$. We use the emcee (Foreman-Mackey et al. 2013) to determine the best value and uncertainty: $K_{1}=24.2 \pm 1.4$ $\mathrm{km} \mathrm{s}^{-1}$ (Figure 2).

\subsection{Lightcurve Modeling}

We modeled the high-cadence lightcurves using the package ellc (Maxted 2016). We use a spherical star to model the white dwarf and use Roche-lobe geometry for the companion. The free parameters for this model are the mid-eclipse time $\left(t_{0}\right)$, inclination ( $i$ ), mass-ratio $(q)$, the radii divided by the semimajor axis of both objects $\left(r_{1,2} \equiv R_{1,2} / a\right)$, the semimajor axis $(a)$, and the surface brightness ratio $\left(J_{g, z}\right)$.

We used a number of fixed parameters in the binary model. First, we use the orbital period obtained from the ZTF data (Section 3.1). For limb-darkening of the white dwarf, we use tabulated values by Claret et al. (2020) for $T=10,000 \mathrm{~K}$ and $\log (g)=8.0$.

In addition, we imposed two restrictions on the white dwarf based on the zero-temperature white dwarf mass-radius relation by Eggleton as reported in Verbunt \& Rappaport (1988). The first is that it cannot be smaller than a zerotemperature white dwarf. The second constraint is a Gaussian prior with a relative size of $5 \%$ compared to a zero-temperature white dwarf to limit the maximum size of the white dwarf. As a 

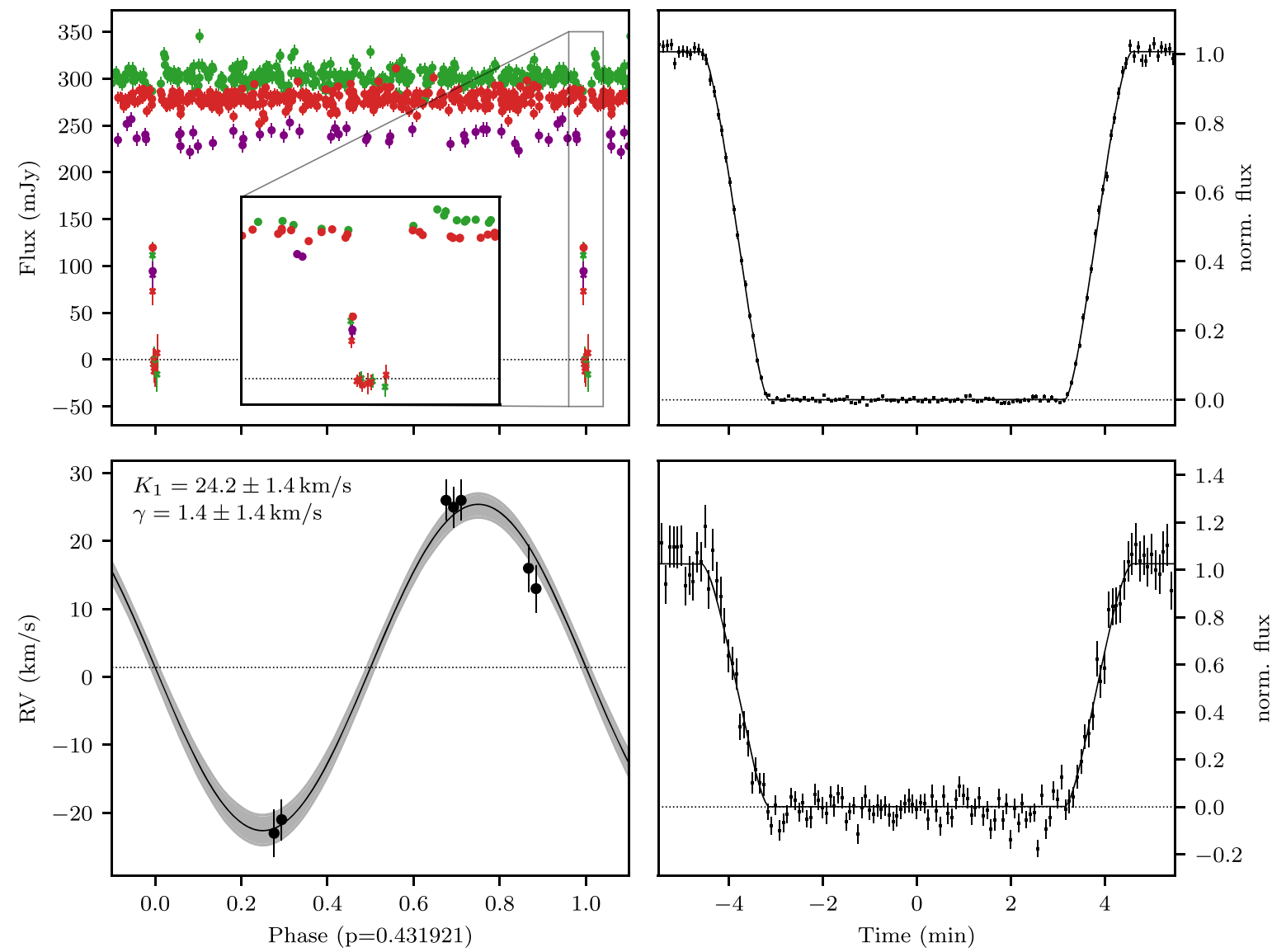

Figure 2. The top left panel shows the ZTF gri data (green, red, purple) folded to the period. The ZTF data shows a deep, narrow eclipse in all three filters. The right two panels show the CHIMERA $g$ (top) and $z$ data (bottom) with the best-fit ellc model overplotted. The bottom left panel shows the ESI radial velocity measurements. The best-fit radial velocity curve is overplotted with the 1-standard deviation in gray and the dotted line indicates the systematic velocity. We modeled the CHIMERA lightcurves combined with the measured radial velocity to measure the system parameters; see Section 3.4.

final constraint, we use a Gaussian prior on the radial velocity amplitude $\left(K_{1}\right)$ of the white dwarf (see Section 3.3).

To find the most probable parameter values and uncertainties, we again use emcee.

\section{Results}

We measured the binary properties by analyzing ZTF lightcurves (Section 3.1, used to determine the orbital period), the spectral energy distribution (Section 3.2, used to derive the temperatures), phase-resolved spectroscopy (Section 3.3, used to measure the white dwarf radial velocity semi-amplitude) and high-cadence $g$ - and $z$-band lightcurves (used to measure the mass and radii of both components). The results are summarized in Table 3 and the posterior of the lightcurve modeling is shown in the Appendix.

The mass of the companion, which is mostly set by the radial velocity semi-amplitude measurement, is $M_{2}=0.0593 \pm 0.004$ $M_{\odot}$, and a radius of $R_{2}=0.0783 \pm 0.0012 R_{\odot}$. The $z$-band surface brightness ratio limits the temperature of the companion to $\lesssim 1550 \mathrm{~K}$ and the spectral energy distribution constrains the temperature further to $\lesssim 750 \mathrm{~K}$.

The mass of the white dwarf is $0.50 \pm 0.02 M_{\odot}$ and the radius is $R_{1}=0.01429 \pm 0.00020 R_{\odot}$. This is consistent with the white dwarf $\mathrm{M}-\mathrm{R}$ relation, which is what we enforced using a prior. The temperature of the white dwarf is $T_{1}=10,900 \pm$ $200 \mathrm{~K}$ and a surface gravity is $\log g=7.83 \pm 0.01$. This is slightly different than reported by Gentile Fusillo et al. (2019)
$(10,290 \pm 210 \mathrm{~K}, \log g=7.94 \pm 0.07)$ but is within two standard deviations.

The orbital separation of the binary system is $a=$ $1.987 \pm 0.027 R_{\odot}$ and the inclination of this system is $i=$ $89^{\circ} .71 \pm 0.13$.

\section{Discussion}

\subsection{The Nature of the Substellar Companion}

In Figure 3, we plot the mass and radius of the companion and compare it to models by Marley et al. (2018) and other binaries with substellar objects. The measured mass and radius agree with models of $10 \mathrm{Gyr}$ old brown dwarfs with $Z \gtrsim 0$ abundances. The models predict a temperature of $\sim 800 \mathrm{~K}$, which is consistent with the limit we derived from the SED, $\lesssim 750 \mathrm{~K}$ (see Figure 1). If we assume a solar abundance or lower, the age of the brown dwarf (and therefore the system) is $\gtrsim 8 \mathrm{Gyr}$.

The initial to final mass relation for the white dwarfs suggests that the white dwarf progenitor was approximately a $1-2 M_{\odot}$ main-sequence star. This corresponds to a mainsequence lifetime of 2-10 Gyr (Catalán et al. 2008; Marigo 2013; Cummings et al. 2018). The cooling age of the white dwarf is approximately $\sim 400 \mathrm{Myr}$ (Koester 2009). Given the age of the brown dwarf, the white dwarf progenitor mass was likely closer to the lower bound of the mass range, $\approx 1 M_{\odot}$. 
Table 3

Binary Parameters Determined by Modeling the Lightcurves using ellc and Modeling the SED

\begin{tabular}{|c|c|}
\hline$\overline{p^{f}(\mathrm{~d})}$ & $0.4319208(14)$ \\
\hline$t_{0}\left(\mathrm{BJD}_{\mathrm{TBD}}\right)$ & $2,459,045.985194(2)$ \\
\hline$q$ & $0.1167_{-0.0068}^{+0.0075}$ \\
\hline$i\left(^{\circ}\right)$ & $89.71_{-0.13}^{+0.12}$ \\
\hline$r_{1}$ & $0.007195_{-0.000078}^{+0.000075}$ \\
\hline$r_{2}$ & $0.03934_{-0.00019}^{+0.00033}$ \\
\hline$a\left(R_{\odot}\right)$ & $1.987_{-0.022}^{+0.030}$ \\
\hline$J_{g}$ & $\lesssim 0.000035$ \\
\hline$J_{z}$ & $\lesssim 0.00014$ \\
\hline$M_{1}\left(M_{\odot}\right)$ & $0.505_{-0.018}^{+0.024}$ \\
\hline$M_{2}\left(M_{\odot}\right)$ & $0.0593_{-0.0039}^{+0.0036}$ \\
\hline $\mathrm{R}_{1}^{p}\left(R_{\odot}\right)$ & $0.01429_{-0.00017}^{+0.00022}$ \\
\hline$R_{2}\left(R_{\odot}\right)$ & $0.0783_{-0.0011}^{+0.0011}$ \\
\hline $\log \left(g_{1}\right)(\operatorname{cgs})$ & $7.832_{-0.013}^{+0.013}$ \\
\hline $\log \left(g_{2}\right)(\operatorname{cgs})$ & $5.425_{-0.03}^{+0.02}$ \\
\hline $\mathrm{K}_{1}^{p}\left(\mathrm{~km} \mathrm{~s}^{-1}\right)$ & $24.4_{-1.4}^{+1.4}$ \\
\hline$K_{2}\left(\mathrm{~km} \mathrm{~s}^{-1}\right)$ & $208.4_{-2.9}^{+3.7}$ \\
\hline $\bar{\rho}_{2}\left(\mathrm{~g} \mathrm{~cm}^{-3}\right)$ & $174_{-11}^{+9}$ \\
\hline $\mathrm{K}_{1}^{p}\left(\mathrm{~km} \mathrm{~s}^{-1}\right)$ & $24.4_{-1.4}^{+1.4}$ \\
\hline$K_{2}\left(\mathrm{~km} \mathrm{~s}^{-1}\right)$ & $208.4_{-2.9}^{+3.7}$ \\
\hline$T_{1}(\mathrm{~K})$ & $10900 \pm 200$ \\
\hline$T_{2}(\mathrm{~K})$ & $\lesssim 750$ \\
\hline distance $(\mathrm{pc})$ & $139 \pm 2$ \\
\hline
\end{tabular}

Note. The top section lists ellc model parameters; the middle section shows the binary parameters derived from the ellc fit. We fixed the orbital period $\left({ }^{f}\right)$ and for the radius of the white dwarf $\left(R_{1}\right)$ and radial velocity amplitude $\left(K_{1}\right)$ we used a prior $\left({ }^{p}\right)$. The temperatures, in the bottom section of the table, have been determined by modeling the SED. We use the $95 \%$ percentile to determine upper limits.

Compared to other substellar objects that are eclipsing white dwarfs, the mass and radius do not stand out and are similar to other brown dwarfs. This object does stand out because of its orbital period, which at $10 \mathrm{hr}$ is an order of magnitude larger than the three other known brown dwarfs orbiting white dwarfs. This means that the amount of irradiation by the white dwarf is relatively low. Using a simple blackbody approximation (Littlefair et al. 2014), we estimate that the temperature of the brown dwarf is only increased by $\sim 50 \mathrm{~K}$ due to irradiation by the white dwarf. This fact, and the system's relative brightness, make it a good prototype system for longperiod white-dwarf-brown-dwarf systems.

\subsection{Formation History}

Since the companion is a brown dwarf and not a giant planet, standard common-envelope evolution can explain the formation of this system. Given that the mass of the white dwarf is $\geqslant 0.47 \mathrm{M}_{\odot}$, the white dwarf very likely has a CO core (see, e.g., Marigo 2013 and also Parsons et al. 2017b for observational evidence) which allows for two formation scenarios. In the first scenario, the white dwarf could have formed during a commonenvelope phase on the AGB after helium-core exhaustion. The second scenario is that the common envelope happened at the tip of the RGB, just after the helium flash (Han et al. 2003) which would result in a white dwarf with a mass close to $0.47 M_{\odot}$. In that scenario the white dwarf would have emerged from the common envelope as a hot subdwarf $(\mathrm{sdB})$ and appeared as an HW Vir system before it evolved into a white dwarf with a brown dwarf companion after helium exhaustion in the $\mathrm{sdB}$. Several $\mathrm{sdB}+$ brown dwarf systems are known, although typically seen with shorter orbital periods (e.g., Geier et al. 2011; Schaffenroth et al. 2015, 2018, 2019).

The white dwarf will slowly cool down and the period will slowly decrease due to gravitational wave radiation. It will take $\sim 135 \mathrm{Gyr}$ to reach an orbital period of $\sim 40$ minutes (Rappaport et al. 2021), at which point the white dwarf will be $\sim 1000 \mathrm{~K}$. Roche-lobe overflow will commence and the system becomes a cataclysmic variable (Littlefair et al. 2003). The accretion flow will heat up the white dwarf again while the period increases. This will slowly drain the brown dwarf and the system ends up as a "period-bouncer"; a very low accretionrate $\mathrm{CV}$ with an orbital period of $\approx 90$ minutes (e.g Pala et al. 2018).

\subsection{Implications for Searches for Brown Dwarfs and Giant Planets Orbiting White Dwarfs}

Here, we briefly discuss the detection efficiency of our search and the occurrence rate of white dwarfs with transiting substellar objects. A detailed simulation is beyond the scope of this paper and we limit ourselves to an order of magnitude estimate only.

To find ZTFJ0038+2030 we searched the ZTF lightcurves of the Gaia white dwarf catalog by Gentile Fusillo et al. (2019) which contains 486,641 candidate white dwarfs over the entire sky. There are 129,148 white dwarfs brighter than 20 mag with more than 80 epochs in their ZTF lightcurve. Based on the number of epochs in these lightcurves, we estimate an average recovery efficiency of $15 \%-25 \%$ (the probability of getting $7-5$ in-eclipse points). We note that we did recover the other three known eclipsing WD-BD systems (Figure 3) that show longer eclipse duty cycles and are therefore easier to find than ZTFJ0038+2030.

With the discovery of ZTFJ0038+2030 and the discovery of its planet-candidate (Vanderburg et al. 2020), there are now two known long-period ( $\gtrsim 10 \mathrm{hr}$ ) transiting substellar objects around white dwarfs; the first is most likely a giant planet and the second a brown dwarf. Based on just these two detections of long-period transiting objects $(\gtrsim 10 \mathrm{hr})$, the occurrence rate of planets and brown dwarfs is the same order of magnitude. If we also consider the non-transiting objects, the white-dwarfbrown-dwarf GD1400 and the white-dwarf-planet system WD $1145+017$, we reach a similar conclusion. However, we note that these latter two systems were discovered due to an infrared excess and peculiar white dwarf emission lines, which are both methods that are heavily biased. In order to systematically measure the occurrence rate of objects spanning masses of $\approx 0.01-0.07 M_{\odot}$, a white dwarf transit search is needed. ${ }^{12}$ Such a survey allows us to measure the mass distribution of white dwarf companions and determine which of the formation channels are important in the formation of these objects.

Currently, the ZTF detection efficiency is limited by the number of epochs available per white dwarf. As more epochs are obtained, ZTF will be able to identify narrower eclipses, which means that longer period systems can be identified. Based on the recovery efficiency of ZTFJ0038+2030, we estimate that ZTF will find another 3-6 similar-sized objects at

\footnotetext{
${ }^{12}$ A large-scale, precise $\left(\lesssim 1 \mathrm{~km} \mathrm{~s}^{-1}\right)$ spectroscopic survey to find radial velocity changes of white dwarfs would also work.
} 


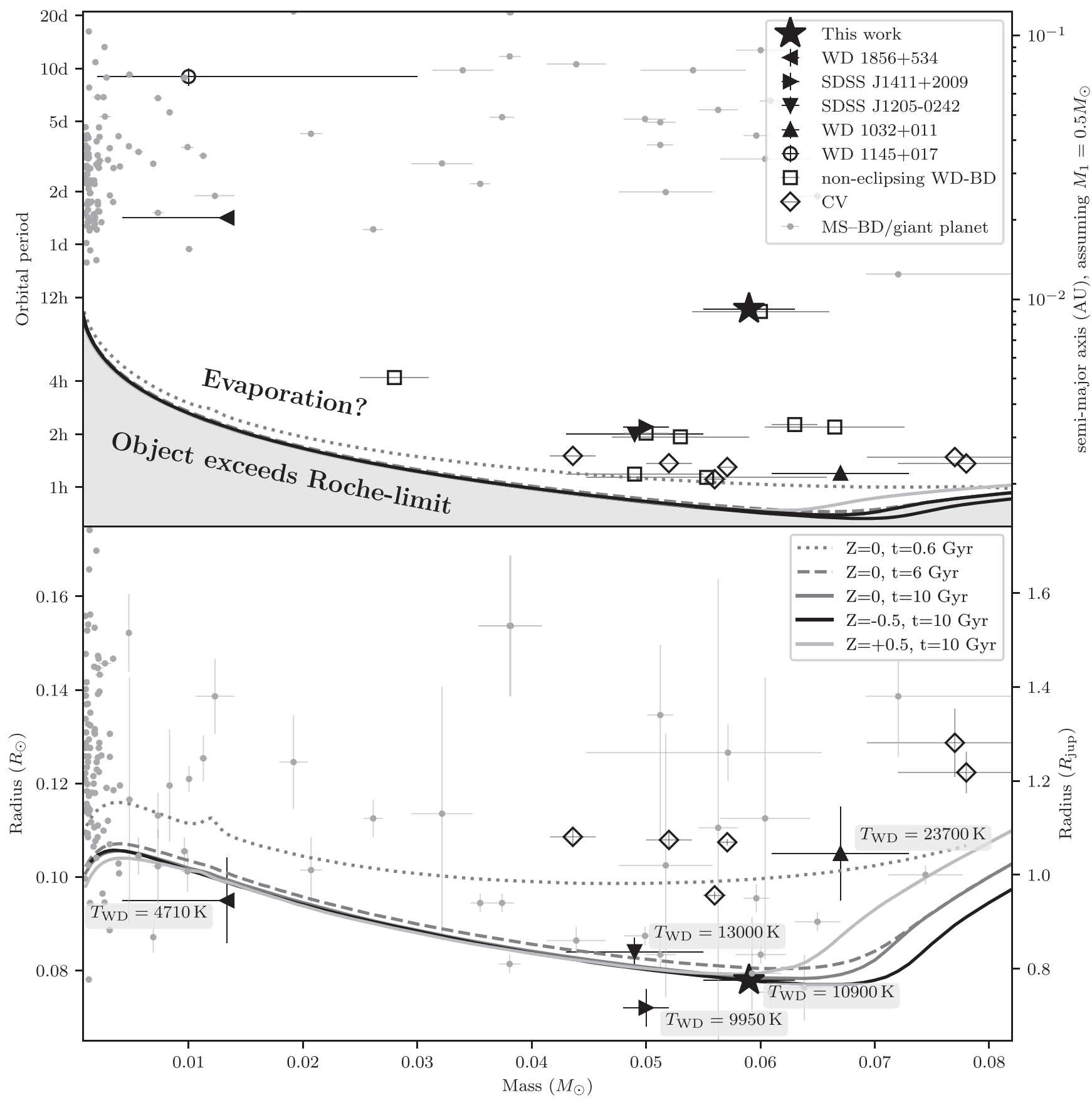

Figure 3. The characteristics of substellar objects that are orbiting white dwarfs. Filled black markers show eclipsing systems, with ZTFJ0038+2030 as a star and other eclipsing systems as triangles (Littlefair et al. 2014; Parsons et al. 2018; Vanderburg et al. 2020; Casewell et al. 2020a). The open circle shows the non-eclipsing WD-planet system from Gänsicke et al. (2019). Open squares show known non-eclipsing white dwarfs with substellar companions (see Section 1). Of note is GD1400 (Farihi \& Christopher 2004; Burleigh et al. 2011), a non-eclipsing system that has almost the same white dwarf mass and orbital period as ZTFJ0038+2030. Open diamonds show cataclysmic variables with low-mass donors (Longstaff et al. 2019). Grey dots show brown dwarfs and giant planets that orbit main-sequence stars from Carmichael et al. (2021) and Chen \& Kipping (2017). Top panel: the orbital period versus the mass. It shows that the known brown dwarfs orbiting white dwarfs are typically found at short orbital periods, close to the Roche limit (Rappaport et al. 2013), while substellar objects around main-sequence stars are found at orbital periods of $\gtrsim 1$ day. Bottom panel: the radius versus the companion mass. The white dwarf temperature is indicated next to each marker. Models are taken from Marley et al. (2018). Young or heavily irradiated substellar objects tend to be larger compared to old and non-irradiated objects (Casewell et al. 2020b). Uncertainties for lowmass objects $\left(M<0.005 M_{\odot}\right)$ are omitted for clarity.

long orbital periods ( $\gtrsim 10 \mathrm{hr})$ as it keeps accumulating more data. Other surveys like Gaia (Gaia Collaboration et al. 2016), ATLAS (Tonry et al. 2018), and BlackGEM (Groot 2019) can be used to find similar systems over the whole sky. In the near future, the Vera C. Rubin observatory (Ivezic̀ et al. 2019) is expected to find many white dwarfs with exoplanets, possibly down to Earth-sized objects (Agol 2011).

\section{Summary and Outlook}

Using ZTF photometry and Gaia and Pan-STARRS data, we discovered an eclipsing binary composed of a white dwarf and a substellar companion with an orbital period of $10 \mathrm{hr}$. We obtained follow-up photometry and spectroscopy and measured the binary parameters. We showed that the substellar 
companion is a $\gtrsim 8 \mathrm{Gyr}$ old, small brown dwarf with a mass of $0.06 M_{\odot}$, and the white dwarf a $0.50 M_{\odot}, \mathrm{CO}$ white dwarf. ZTFJ0038+2030 has a much longer orbital period than most known white-dwarf-brown-dwarf systems, and is very similar to non-transiting white-dwarf-brown-dwarf GD1400. However, GD1400 shows an infrared excess (which is how it was discovered) while ZTFJ0038+2030 does not because the brown dwarf is older and has a lower temperature.

ZTFJ0038+2030 is relatively bright and because the brown dwarf suffers minimal irradiation, it can be used to study the brown dwarf atmosphere, while the mass and radius can be measured even more precisely by obtaining additional highspeed photometry and phase-resolved spectra. It is also a useful target for eclipse timing to find circumbinary objects (e.g., NN Ser, Marsh et al. 2014) as brown dwarfs are not expected to show eclipse time variations due to Applegate's mechanism (Applegate 1992; Bours et al. 2016). The discovery of this system demonstrates that ZTF data can be used to find substellar objects in long periods orbiting white dwarfs and we expect ZTF to find more of these systems as more data are obtained.

We thank the anonymous referee for detailed and constructive feedback.

J.vR. is partially supported by NASA-LISA grant 76280NSSC19K0325. K.J.B. is supported by the National Science Foundation under Award AST-1903828.

We thank Lars Bildsten for trading one hour of ESIKeck time.

Based on observations obtained with the Samuel Oschin 48 inch Telescope and the 60 inch Telescope at the Palomar Observatory as part of the Zwicky Transient Facility project. ZTF is supported by the National Science Foundation under grant No. AST-1440341 and a collaboration including Caltech, IPAC, the Weizmann Institute for Science, the Oskar Klein
Center at Stockholm University, the University of Maryland, the University of Washington, Deutsches Elektronen-Synchrotron and Humboldt University, Los Alamos National Laboratories, the TANGO Consortium of Taiwan, the University of Wisconsin at Milwaukee, and Lawrence Berkeley National Laboratories. Operations are conducted by COO, IPAC, and UW.

Based on observations obtained with the 200 inch Hale Telescope at the Palomar Observatory as part of the Zwicky Transient Facility project. The Hale telescope is operated by the Caltech Optical Observatories.

Some of the data presented herein were obtained at the W.M. Keck Observatory, which is operated as a scientific partnership among the California Institute of Technology, the University of California, and the National Aeronautics and Space Administration. The Observatory was made possible by the generous financial support of the W.M. Keck Foundation. The authors wish to recognize and acknowledge the very significant cultural role and reverence that the summit of Maunakea has always had within the indigenous Hawaiian community. We are most fortunate to have the opportunity to conduct observations from this mountain.

Facilities: PO:1.2 m (ZTF), Hale (CHIMERA), Keck:II (ESI).

Software: VizieR, astropy (Astropy Collaboration et al. 2013), NumPy (Harris et al. 2020), SciPy (Virtanen et al. 2020), matplotlib (Hunter 2007), Makee, ellc (Maxted et al. 2014), emcee (Foreman-Mackey et al. 2013).

\section{Appendix Appendix Information}

Figure 4 shows the posterior distribution of the Chimera lightcurve modelling. 


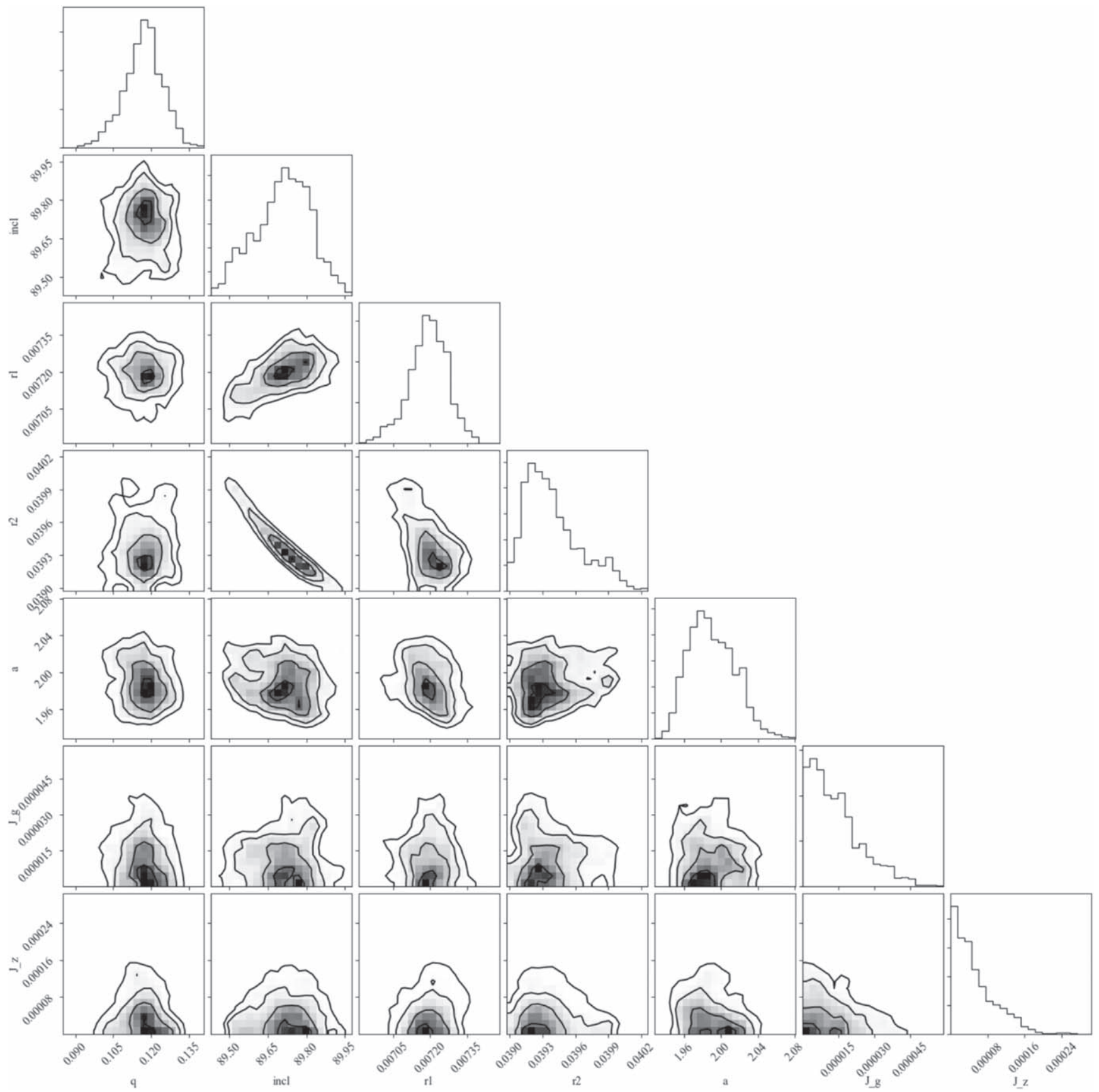

Figure 4. The posterior distribution of the fit to the lightcurve data using ellc and emcee.

\section{ORCID iDs}

Jan van Roestel (iD https://orcid.org/0000-0002-2626-2872

Thomas Kupfer (iD https://orcid.org/0000-0002-6540-1484

Keaton J. Bell (i) https://orcid.org/0000-0002-0656-032X

Kevin Burdge (iD https://orcid.org/0000-0002-7226-836X

Przemek Mróz (1) https://orcid.org/0000-0001-7016-1692

Thomas A. Prince (i) https://orcid.org/0000-0002-8850-3627

Eric C. Bellm (i) https://orcid.org/0000-0001-8018-5348

Richard Dekany (i) https://orcid.org/0000-0002-5884-7867

Ashish A. Mahabal (i) https://orcid.org/0000-0003-2242-0244

Reed Riddle (iD https://orcid.org/0000-0002-0387-370X

Kyung Min Shin (i) https://orcid.org/0000-0002-1486-3582

David L. Shupe (ib https://orcid.org/0000-0003-4401-0430

S. R. Kulkarni (1) https://orcid.org/0000-0001-5390-8563

\section{References}

Agol, E. 2011, ApJL, 731, L31

Applegate, J. H. 1992, ApJ, 385, 621

Astropy Collaboration, Robitaille, T. P., \& Tollerud, E. J. 2013, A\&A, 558, A33

Bailer-Jones, C. A. L., Rybizki, J., Fouesneau, M., Demleitner, M., \& Andrae, R. 2021, AJ, 161, 147

Bear, E., \& Soker, N. 2011, MNRAS, 414, 1788

Bellm, E., Kulkarni, S., \& Graham, M. 2019, AAS Meeting, 233, 363.08

Beuermann, K., Dreizler, S., Hessman, F. V., et al. 2013, A\&A, 558, A96

Bianchi, L., Shiao, B., \& Thilker, D. 2017, ApJS, 230, 24

Bours, M. C. P., Marsh, T. R., Parsons, S. G., et al. 2016, MNRAS, 460, 3873

Brown, A. G. A., Vallenari, A., Prusti, T., et al. 2020a, A\&A, 649, A1

Brown, W. R., Kilic, M., Bédard, A., Kosakowski, A., \& Bergeron, P. 2020b, ApJL, 892, L35

Burleigh, M. R., Marsh, T. R., Gänsicke, B. T., et al. 2006, MNRAS, 373,1416 
Burleigh, M. R., Steele, P. R., Dobbie, P. D., et al. 2011, in AIP Conf. Proc. 1331, Planetary Systems Beyond the Main Sequence, ed. S. Schuh, H. Dreschsel, \& U. Heber, 262

Carmichael, T. W., Quinn, S. N., Zhou, G., et al. 2021, AJ, 161, 97

Casewell, S. L., Belardi, C., Parsons, S. G., et al. 2020a, MNRAS, 497, 3571

Casewell, S. L., Burleigh, M. R., Wynn, G. A., et al. 2012, ApJL, 759, L34

Casewell, S. L., Debes, J., Braker, I. P., et al. 2020b, MNRAS, 499, 5318

Casewell, S. L., Lawrie, K. A., Maxted, P. F. L., et al. 2015, MNRAS, 447, 3218

Casewell, S. L., Littlefair, S. P., Parsons, S. G., et al. 2018, MNRAS, 481, 5216

Catalán, S., Isern, J., García-Berro, E., \& Ribas, I. 2008, MNRAS, 387, 1693

Chambers, K. C., Magnier, E. A., Metcalfe, N., et al. 2016, arXiv:1612.05560

Chen, J., \& Kipping, D. 2017, ApJ, 834, 17

Claret, A., Cukanovaite, E., Burdge, K., et al. 2020, A\&A, 634, A93

Cummings, J. D., Kalirai, J. S., Tremblay, P.-E., Ramirez-Ruiz, E., \& Choi, J. 2018, ApJ, 866, 21

Dame, K., Belardi, C., Kilic, M., et al. 2019, MNRAS, 490, 1066

Dekany, R., Smith, R. M., Riddle, R., et al. 2020, PASP, 132, 038001

Dhillon, V. S., Marsh, T. R., Stevenson, M. J., et al. 2007, MNRAS, 378, 825

Dobbie, P. D., Barstow, M. A., Hubeny, I., et al. 2005, MNRAS, 363, 763

Dye, S., Lawrence, A., Read, M. A., et al. 2018, MNRAS, 473, 5113

Faedi, F., West, R. G., Burleigh, M. R., Goad, M. R., \& Hebb, L. 2011, MNRAS, 410, 899

Farihi, J., \& Christopher, M. 2004, AJ, 128, 1868

Farihi, J., Parsons, S. G., \& Gänsicke, B. T. 2017, NatAs, 1, 0032

Fitzpatrick, E. L. 1999, PASP, 111, 63

Foreman-Mackey, D., Hogg, D. W., Lang, D., \& Goodman, J. 2013, PASP, 125,306

Fulton, B. J., Tonry, J. L., Flewelling, H., et al. 2014, ApJ, 796, 114

Gaia Collaboration, Prusti, T., de Bruijne, J. H. J., et al. 2016, A\&A, 595, A1

Gänsicke, B. T., Schreiber, M. R., Toloza, O., et al. 2019, Natur, 576, 61

Geier, S., Heber, U., Tillich, A., et al. 2011, in AIP Conf. Proc. 1331, Planetary Systems Beyond the Main Sequence, ed. S. Schuh, H. Dreschsel, \& U. Heber, 163

Gentile Fusillo, N. P., Tremblay, P.-E., Gänsicke, B. T., et al. 2019, MNRAS, 482,4570

Graham, M. J., Kulkarni, S. R., Bellm, E. C., et al. 2019, PASP, 131, 078001

Green, M. J., Marsh, T. R., Steeghs, D. T. H., et al. 2018, MNRAS, 476, 1663

Groot, P. J. 2019, NatAs, 3, 1160

Han, Z., Podsiadlowski, P., Maxted, P. F. L., \& Marsh, T. R. 2003, MNRAS, 341, 669

Harding, L. K., Hallinan, G., Milburn, J., et al. 2016, MNRAS, 457, 3036

Harris, C. R., Millman, K. J., van der Walt, S. J., et al. 2020, Natur, 585, 357

Hunter, J. D. 2007, CSE, 9, 90

Ivanova, N., Justham, S., Chen, X., et al. 2013, A\&ARv, 21, 59

Ivezic̀, v., Kahn, S. M., Tyson, J. A., et al. 2019, ApJ, 873, 111

Koester, D. 2009, A\&A, 498, 517

Kovács, G., Zucker, S., \& Mazeh, T. 2002, A\&A, 391, 369

Kupfer, T., Bauer, E. B., Marsh, T. R., et al. 2020, ApJ, 891, 45

Kupfer, T., Ramsay, G., Roestel, J. v., et al. 2017b, ApJ, 851, 28

Kupfer, T., van Roestel, J., Brooks, J., et al. 2017a, ApJ, 835, 131
Lagos, F., Schreiber, M. R., Zorotovic, M., et al. 2021, MNRAS, 501, 676

Littlefair, S. P., Casewell, S. L., Parsons, S. G., et al. 2014, MNRAS, 445, 2106

Littlefair, S. P., Dhillon, V. S., \& Martín, E. L. 2003, MNRAS, 340, 264

Longstaff, E. S., Casewell, S. L., Wynn, G. A., et al. 2019, MNRAS, 484, 2566

Marigo, P. 2013, in IAU Symp. 281, Asymptotic Giant Branch Evolution and the Initial-Final Mass Relation of Single $\mathrm{CO}$ White Dwarfs, ed. R. Di Stefano, M. Orio, \& M. Moe, 36

Marley, M., Saumon, D., Morley, C., \& Fortney, J. 2018, Sonora 2018: Cloudfree, solar composition, solar C/O substellar atmosphere models and spectra v.nc_m+0.0_co1.0_v1.0, Zenodo, doi:10.5281/zenodo.1309035

Marocco, F., Eisenhardt, P. R. M., Fowler, J. W., et al. 2021, ApJS, 253, 8

Marsh, T. R., Parsons, S. G., Bours, M. C. P., et al. 2014, MNRAS, 437, 475

Masci, F. J., Laher, R. R., Rusholme, B., et al. 2019, PASP, 131, 018003

Maxted, P. F. L. 2016, A\&A, 591, A111

Maxted, P. F. L., Napiwotzki, R., Dobbie, P. D., \& Burleigh, M. R. 2006, Natur, 442, 543

Maxted, P. F. L., Serenelli, A. M., Marsh, T. R., et al. 2014, MNRAS, 444, 208

Napiwotzki, R., Karl, C. A., Lisker, T., et al. 2004, Ap\&SS, 291, 321

Nelemans, G., \& Tauris, T. M. 1998, A\&A, 335, L85

Pala, A. F., Schmidtobreick, L., Tappert, C., Gänsicke, B. T., \& Mehner, A 2018, MNRAS, 481, 2523

Parsons, S. G., Gänsicke, B. T., Marsh, T. R., et al. 2017b, MNRAS, 470, 4473

Parsons, S. G., Gänsicke, B. T., Marsh, T. R., et al. 2018, MNRAS, 481, 1083

Parsons, S. G., Hermes, J. J., Marsh, T. R., et al. 2017a, MNRAS, 471, 976

Perets, H. B. 2010, arXiv:1001.0581

Phillips, M. W., Tremblin, P., Baraffe, I., et al. 2020, A\&A, 637, A38

Rappaport, S., Sanchis-Ojeda, R., Rogers, L. A., Levine, A., \& Winn, J. N. 2013, ApJL, 773, L15

Rappaport, S., Vanderburg, A., Schwab, J., \& Nelson, L. 2021, ApJ, 913, 118

Rodrigo, C., \& Solano, E. 2020, in Contributions to the XIV.0 Scientific Meeting (virtual) of the Spanish Astronomical Society, The SVO Filter Profile Service, 182

Rodrigo, C., Solano, E., Bayo, A., \& Rodrigo, C. 2012, IVOA Working Draft 15 October 2012

Rowan, D. M., Tucker, M. A., Shappee, B. J., \& Hermes, J. J. 2019, MNRAS, 486, 4574

Schaffenroth, V., Barlow, B. N., Drechsel, H., \& Dunlap, B. H. 2015, A\&A, 576, A123

Schaffenroth, V., Barlow, B. N., Geier, S., et al. 2019, A\&A, 630, A80

Schaffenroth, V., Geier, S., Heber, U., et al. 2018, A\&A, 614, A77

Sheinis, A. I., Bolte, M., Epps, H. W., et al. 2002, PASP, 114, 851

Soker, N. 1998, AJ, 116, 1308

Steele, P. R., Saglia, R. P., \& Burleigh, M. R. 2013, MNRAS, 429, 3492

Stephan, A. P., Naoz, S., \& Gaudi, B. S. 2020, arXiv:2010.10534

Tonry, J. L., Denneau, L., Heinze, A. N., et al. 2018, PASP, 130, 064505

van Roestel, J., Creter, L., Kupfer, T., et al. 2021, ApJ, 162, 113

van Sluijs, L., \& Van Eylen, V. 2018, MNRAS, 474, 4603

Vanderburg, A., Rappaport, S. A., Xu, S., et al. 2020, Natur, 585, 363

Verbunt, F., \& Rappaport, S. 1988, ApJ, 332, 193

Virtanen, P., Gommers, R., Oliphant, T. E., et al. 2020, Nat. Methods, 17, 261 